\title{
LHomme
}

L'HOMME Revue française d'anthropologie

225 | 2018

Varia

\section{Le structuralisme renouvelé de Françoise Héritier}

\section{Enric Porqueres i Gené}

\section{OpenEdition}

Journals

Édition électronique

URL : http://journals.openedition.org/lhomme/30719

DOI : ERREUR PDO dans /localdata/www-bin/Core/Core/Db/Db.class.php L.34 : SQLSTATE[HY000]

[2006] MySQL server has gone away

ISSN : 1953-8103

\section{Éditeur}

Éditions de l'EHESS

Édition imprimée

Date de publication : 15 mars 2018

Pagination : 15-22

ISBN : 978-2-7132-2733-2

ISSN : 0439-4216

\section{Référence électronique}

Enric Porqueres i Gené, «Le structuralisme renouvelé de Françoise Héritier » L'Homme [En ligne], 225 | 2018, mis en ligne le 15 mars 2018, consulté le 08 janvier 2021. URL : http://journals.openedition.org/ Ihomme/30719 ; DOI : https://doi.org/ERREUR PDO dans /localdata/www-bin/Core/Core/Db/ Db.class.php L.34 : SQLSTATE[HY000] [2006] MySQL server has gone away 



\title{
Le structuralisme renouvelé de Françoise Héritier
}

\author{
Enric Porqueres i Gené
}

T

RÈS TÔT, j'ai été impressionné par l'exigence et l'insatiable curiosité intellectuelles qui animaient cette chercheuse d'exception qu'était Françoise Héritier. Un souvenir, en particulier, me revient en mémoire. À l'occasion de ma soutenance de thèse en 1994, nous nous trouvions à Florence et déambulions dans la ville à la découverte des chefs-d'œuvre de la Renaissance italienne qui s'offraient à nos yeux. Face à cette riche iconographie aux significations complexes qui nous échappaient en grande partie, c'est avec un enthousiasme redoublé qu'elle multipliait les questions pour décrypter les représentations mettant en scène des personnages mythologiques ou bibliques: de l'ordonnancement en pyramide de la fresque d'Andrea del Sarto illustrant la Vierge au sac (Madonna del sacco) sur un tympan de la basilique della Santissima Annunziata, à la figura serpentinata et aux couleurs crues de l'Annonciation et de la Déposition du peintre maniériste Pontormo dans l'église Santa Felicità, la passion du détail accompagnait à tout moment l'expérience esthétique. Si je pense ici à cet épisode florentin, maintes fois évoqué par ailleurs dans nos conversations, c'est que, pour moi, il est particulièrement illustratif de la méthode de Françoise Héritier, combinant finesse de l'analyse et vision d'ensemble pour former "une pensée en mouvement ».

À condition de bien en distinguer les composantes, l'œuvre de Françoise Héritier tient en une série de propositions théoriques qui ont contribué à une compréhension plus subtile du phénomène de la parenté. Opérant un tournant empiriste - qu'elle préférait qualifier de matérialiste -, elle donne un nouveau fondement à des éléments centraux de la démarche structuraliste. Bien des pistes de cette avancée restent encore à explorer, ce qui démontre la puissance d'une réflexion souvent comprise de façon partielle. Parce qu'il bouleverse la division traditionnellement établie entre sociologie de la famille (dans les sociétés individualistes) et anthropologie de la parenté et de la personne relationnelle (dans les sociétés exotiques), le travail de Françoise 
Héritier permet de situer la parenté et ses principes au centre de toutes les constructions sociales, y compris celles dites «contemporaines». Ajoutons que cette contribution s'est construite dans un contexte de crise épistémologique.

À l'époque où Françoise Héritier publie L'Exercice de la parenté (1981), en effet, certains spécialistes anglo-saxons des sociétés pratiquant l'échange généralisé, s'étant pourtant saisis des analyses structurales, comme Edmund Leach (1961) et Rodney Needham (1971) remettent en cause la sous-discipline traitant de la parenté: ils dénoncent l'application abusive à des sociétés autres de catégories de la parenté empruntées à la culture occidentale. Cette voie critique, poussée à l'extrême par l'anthropologue David Schneider (1984), qui conteste la notion de généalogie et sa centralité dans la construction de l'objet parenté - ce qu'il appelle l'«unité généalogique de l'humanité»-, connaît, en France, des développements particulièrement significatifs. D'un côté, le procès de la méthode généalogique fait par Pierre Bourdieu (1972) qui, à partir de ses enquêtes sur la société kabyle, envisage les généalogies non pas comme des descriptions objectives de ce qui organise un système de parenté, disponibles telles quelles pour l'ethnologue, mais comme des entités inscrites dans le contexte social de leur production, donc sujettes à manipulations et à interprétations. De l'autre, les observations de Claude Lévi-Strauss à propos des sociétés à maison (1975), montrent que, même si le système de parenté continue d'y exprimer l'ordre social, il n'y serait plus qu'un langage justificatif, une sorte d'idéologie masquant les véritables dynamiques sociales de nature économique ou politique. Le maître du structuralisme, s'éloignant progressivement de l'analyse de la règle matrimoniale, emploie alors les notions de stratégie et de tactique, et parle de "fétichisme de la parenté» pour décrire ce qui apparaît in fine comme un stade évolutif particulier, celui dans lequel la parenté, tout en gardant une centralité idéologique, a cessé d'être un facteur structurant de l'ordre social.

Sans se positionner ouvertement sur les questions soulevées par ses collègues, Françoise Héritier met en place une double grille d'analyse. Celle-ci résulte de son adhésion aux théories structuralistes, notamment au cadre programmatique proposé par Claude Lévi-Strauss dans l'introduction à la deuxième édition des Structures élémentaires de la parenté (1967), ainsi que de ses travaux sur la notion de personne chez les Samo de HauteVolta (Héritier 1973), l'actuel Burkina Faso, qui, marqués par la tradition française de recherche ethnologique sur l'Afrique de l'Ouest, conferent une importance centrale à l'analyse des symboles concrets ${ }^{1}$ déterminant les

1. Nous avons organisé, avec Cécile Gribomont et Jérôme Wilgaux, un numéro de la revue Incidence autour de l'anthropologie symbolique de Françoise Héritier (Gribomont, Porqueres i Gené \& Wilgaux 2013). Le lecteur trouvera dans ma contribution à ce numéro une tentative de situer son travail dans l'histoire de la discipline. 
modalités de l'appartenance: à l'humanité d'abord, aux réseaux de parenté ensuite. La combinaison de l'approche structuraliste avec une ethnographie particulièrement attentive aux logiques matérielles des symboles aboutit à leur dépassement, redéfinissant à chaque fois les champs de recherche dans lesquels ces deux traditions s'inscrivent. De sorte que, tout en restant éloignée des débats entre rationalité et relativisme qui ont fait rage pendant les années 1960 et 1970 chez les Anglo-Saxons, Françoise Héritier inaugure un nouveau paradigme pour comprendre le social, et donc la parenté.

Dévoiler les continuités entre les différents types de systèmes de parenté, tel est l'un des objectifs premiers de Françoise Héritier. Pour un anthropologue imprégné de structuralisme lévi-straussien, ces continuités passent tout d'abord par l'alliance et les modalités de l'échange. Ainsi, notamment dans L'Exercice de la parenté, elle scrute les trois types de systèmes d'alliance identifiés par Lévi-Strauss, du moins à partir de "The Future of Kinship Studies» (1965): les structures élémentaires de la parenté, semi-complexes puis complexes. Elle se donne pour tâche de découvrir les constantes qui sous-tendent et permettent de mieux comprendre la variété des cultures humaines. L'unité psychique de l'homme étant l'un des principes de base retenus par ce paradigme, le questionnement de Françoise Héritier s'insère bien dans la logique de l'école de pensée dont elle s'est toujours revendiquée. Or la recherche menée, alors qu'elle vient parfaire l'exercice comparatif structuraliste, opère une transformation d'envergure.

Certes, dans une bonne partie de L'Exercice de la parenté, la logique de l'échange articulée autour des groupes de descendance est mise en avant. C'est ce que rappellent les fameux bouclages consanguins, qui établissent des alliances matrimoniales là où les interdits cessent d'exister: en pays samo, dans les lignages de la mère du père du père, de la mère de la mère du père, de la mère de la mère de la mère et dans celui de la mère du père de la mère. Cependant, ce constat ne se fait plus à partir de l'étude des seules normes matrimoniales. Deux éléments nouveaux interviennent pour donner une tournure particulière à l'analyse: d'une part, la prise en compte des représentations natives de ce qui fait un bon mariage - dans le cas samo, l'équilibre et la compatibilité nécessaires entre les sangs du mari et de l'épouse, transmis à leur descendance -; d'autre part, la restitution d'un nombre important de généalogies recueillies sur le terrain, puis leur traitement informatique ${ }^{2}$.

En déplaçant l'attention sur ce que les gens font, et non plus ce qu'ils disent qu'il faut faire, il devient dès lors indispensable de procéder à partir de corpus généalogiques afin de démêler les "complexités de l'alliance»,

2. Avec l'aide de Marion Selz, statisticienne et informaticienne. 
comme en témoignent les recherches poursuivies, en France, par Michael Houseman, Laurent Barry et, plus récemment, Klaus Hamberger. La même exigence apparaît dans le cadre des structures complexes de la parenté. Pierre Lamaison (1979) ou encore Gérard Delille (1985) se sont intéressés à la présence des bouclages matrimoniaux dans des contextes en principe dépourvus de groupes de descendance. À l'instar de ce qui se produit dans un système semi-complexe, les mariages ont lieu de préférence là où les interdits tombent. En somme, comme dans les systèmes de parenté à structures élémentaires, dans les systèmes semi-complexes et parfois complexes, l'alliance est déterminée par le(s) groupe(s) de descendance ou par les liens généalogiques reliant Ego à Alter. L'équilibre entre le "pas trop proche» et le "pas trop loin» caractérisera ainsi non seulement les travaux des africanistes, mais aussi ceux des européanistes traitant des sociétés paysannes (Zonabend 1981 ; Segalen 1981; Bestard 1986). La continuité entre les différents systèmes se manifeste donc par la présence de logiques matrimoniales qui considèrent l'alliance en fonction de l'appartenance consanguine. Pourtant, une autre voie d'exploration reste possible.

L'importance accordée aux actes matrimoniaux effectivement réalisés ouvre la discipline à l'histoire liée aux effets structurants des actes matrimoniaux. Claude Lévi-Strauss l'énonçait déjà à propos de la capacité des mariages contractés à créer des liens de parenté dans les régimes semicomplexes. Si le premier structuralisme avait décrété la naturalité des liens de consanguinité, la voie était désormais ouverte pour une déconstruction de cette proposition. J'ai personnellement poursuivi cette autre option qui consiste non pas tant à dégager la logique d'une alliance qui met en relation des groupes préexistants, mais à considérer cette même alliance comme un moteur de définition et de constitution des unités de parenté. Ainsi, j'avais abordé les dynamiques historiques de groupes qui, tout en se donnant pour ciment le langage de la généalogie et ses stabilités, n’en étaient pas moins redéfinis dans leurs contours et leurs contenus par les effets des mariages de ses membres. Ceux-ci, notamment en milieu cognatique, s'érigent en effet en constitutifs des sangs et des généalogies des individus à venir (Porqueres i Gené 1995, 2001) et rendent particulièrement instables et soumises à correction des unités identitaires qui paradoxalement proclament leur pérennité. L'inscription de l'histoire dans la structure constituait l'autre direction possible des recherches après la deuxième édition des Structures élémentaires de la parenté. Et, bien que Françoise Héritier s'était engagée dans la première de ces voies, elle n'avait pourtant pas manqué d'encourager cette autre ligne de recherche dans la belle préface qu'elle avait donnée à mon livre (Héritier 1995). Je lui en serai toujours reconnaissant. 
L'attention portée aux discours et représentations natives sur la parenté a guidé la recherche et les constructions théoriques pendant les dernières décennies. Claude Lévi-Strauss, Edmund Leach ou Rodney Needham avaient prévu cette évolution. Bien sûr, il y avait eu des précédents. L'ancienne distinction de William H. Rivers entre kinship et descent, entre les représentations natives de ce qui fait la proximité entre les êtres apparentés et la logique juridique inscrite dans l'analyse des groupes de descendance qui découpent autrement les appartenances sociales, parcourt l'histoire de la discipline. D'une certaine façon, les moments clés dans l'exploration des logiques autonomes du kinship se trouvent paradoxalement du côté de ceux qui sont allés le plus loin dans l'analyse des groupes de descendance. Entre autres, Monica Wilson, Edward E. Evans-Pritchard ou Meyer Fortes ont consacré de très belles pages à la description de proximités particulièrement significatives qui sont même, parfois, en contradiction avec les régimes de descendance décrits (Porqueres i Gené 2006), montrant par là le caractère autonome d'une série de représentations traditionnellement non convoquées pour rendre compte du fonctionnement des systèmes de parenté.

En partant des représentations et des pratiques natives, le deuxième aspect des recherches de Françoise Héritier se rapporte aux travaux d'Émile Durkheim, qu'elle aimait à citer, plus particulièrement le Durkheim de la maturité, celui des Formes élémentaires de la vie religieuse. Dans cet ouvrage, Durkheim appréhende les liens de parenté d'une façon qui s'inspire largement des débats sur le totémisme qui avaient marqué le tournant du $\mathrm{Xx}^{\mathrm{e}}$ siècle. Il y présente notamment ce qu'il avait appelé le lien sacré de la parenté comme étant, à l'instar des idées, des rituels et des représentations qui le sous-tendent, essentiellement contagieux. À partir d'exemples émanant des sociétés australiennes, il établit que le corps et sa porosité au contact d'autres corps expliquent les affiliations aux divers groupes de parenté. Ceux-ci, tout comme les généalogies mythiques qui les accompagnent et les légitiment, apparaissent alors comme secondaires par rapport à des rituels et des représentations liés au corps. Le travail de Françoise Héritier s'attache justement à poser les bases d'une déconstruction des liens de consanguinité, autrefois naturalisés, qui constitue depuis un des objectifs centraux de la recherche.

C'est donc le corps susceptible de s'altérer et de modifier l'identité de son porteur qui est au centre de ce deuxième volet des recherches de Françoise Héritier. En approfondissant la théorie de l'alliance, elle est amenée à définir l'«inceste du deuxième type», concernant les consanguins des partenaires sexuels qui deviennent des personnes proches d'Ego. L'explication d'ordre symbolique de la prohibition de l'inceste permet de rendre compte de nombreuses autres prohibitions qui n'étaient jusque-là pas problématisées par la théorie anthropologique. Il devient clair par ailleurs que le corps non 
seulement transmet des parcelles d'identité dans la consanguinité: il est à la fois produit et producteur d'actes de rapprochement, éminemment sexuels, qui viennent changer la parentèle d'Ego et aussi celles de ses proches. Ses analyses portant sur les modalités concrètes des mises en contact corporelles susceptibles de modifier l'identité propre constituent ainsi une anthropologie symbolique du corps et de ses fluides qui rend possible une véritable réflexion anthropologique sur la condition humaine. Là aussi, l'ouverture vers l'historicité que Lévi-Strauss situait au centre du développement futur des études sur la parenté apparaît avec force. Ce sont en dernière instance les actes sexuels individuels qui arrêtent les contours des groupes d'appartenance liés à la parenté et qui se traduisent dans des prohibitions sexuelles et matrimoniales, véritable plus petit dénominateur commun des systèmes de parenté. Il est important de le rappeler aujourd'hui, après la parution du livre de Marshall Sahlins (2012) sur la définition de la parenté. Bien qu’une profusion de symboles modèle ici ou là les systèmes de parenté, tous ces symboles n'ont pas la même portée. La récurrence systématique de certains éléments indique leur centralité. Comme Françoise Héritier l'a montré, ces éléments sont aisément identifiables dans les références, positives et surtout négatives, portant sur la sexualité. Ils se réfèrent au corps relationnel. Renouant avec la tradition des études sur la personne et ses composantes, la thématisation du corps jette les fondements pour une approche comparative qui ne se satisfait pas des arguments culturalistes envisageant la parenté comme le corrélat de l'arbitraire linguistique qui définit la vie humaine en société.

Les perspectives offertes par la nouvelle façon de mobiliser la sphère du kinship, soit de la parenté telle qu'elle apparait dans les termes employés par les intéressés eux-mêmes et non pas dans les notions abstraites de consanguinité, d'alliance ou de descendance de la théorie anthropologique, permettent de comprendre autrement les logiques de l'échange. Mais, au-delà de l'application de cette grille de lecture sur les terrains classiques de l'anthropologie, Françoise Héritier ouvre la voie à la prise en compte des questions de société actuelles dans l'étude de la parenté. En témoignent ses travaux sur le sida, mais aussi ceux portant sur la reproduction médicalement assistée. Sur ce dernier sujet, Françoise Héritier trouve des équivalents non modernes aux solutions biotechnologiques: la possibilité de multiplier les pères et mères, la pratique des gestations pour autrui, l'arrêt des filiations alors que géniteur et pater ne sont pas la même personne... Dès lors que la démarche de Françoise Héritier ne se contente pas d'analyser localement, chez les Samo par exemple, comment s'articulent les représentations sur le corps poreux et les prohibitions matrimoniales, moyennant une importante montée en généralité, le désidérata lévi-straussien maintes fois proclamé de rendre compte de la parenté dans tous les contextes sociaux se voit ainsi finalement exaucé. 
Un pas supplémentaire est en effet franchi avec la mise en place d'un système comparatif puissant, qui vise à tester et à affiner les hypothèses qui trouvent leur source dans le travail de terrain. Françoise Héritier s’appuie toujours sur les propos des natifs. Un corpus impressionnant, rassemblant des exemples non seulement ethnographiques, mais également historiques et contemporains, lui permet de poser une série de questions qui bousculent l'état de nos connaissances et élargissent le champ empirique de l'anthropologue. Toujours à partir des observations ethnographiques, Héritier va donner un sens nouveau aux systèmes d'oppositions de Lévi-Strauss. Ce sont des évidences immédiates, qu'il est impossible d'ignorer, qui fournissent désormais les bases de tout système de classification. Ces themata basica, comme elle aimait à les appeler, s'imposent à la perception dans leur logique binaire, mais chaque culture va leur donner des valeurs différentes, les insérant dans des jeux d'opposition qui lui sont propres: le sec et l'humide, le haut et le bas, le chaud et le froid... Ces oppositions de base sont inscrites dans le corps, matière première de la symbolique humaine: l'incontournable différence anatomique entre les sexes constituant en fait le socle des notions d'identique et de différent, nécessaire à tout système de classification. À partir de ce constat, dans sa recherche menée principalement depuis sa chaire au Collège de France ${ }^{3}$, Françoise Héritier explore non seulement les éléments fondateurs de la classification, mais également leur syntaxe et ses contraintes. L'association récurrente de certaines notions, liées par exemple à des fonctions corporelles comme la fécondité ou la digestion, suit une logique de regroupement qui n'apparaît que quand elle est étudiée dans le jeu des variations culturelles sur un même thème. Ce sont les "chaînes de concepts auto-associés", pour reprendre la formule de Françoise Héritier. De la sorte, en reconnaissant des associations nécessaires entre concepts dans l'ensemble des cultures humaines, la frontière généralement établie entre les sociétés dites exotiques, ou holistiques, et celles individualistes, dites contemporaines, perd de sa pertinence. En effet, l'anthropologie symbolique du corps développée par Françoise Héritier permet de dépasser la traditionnelle distinction entre «l'Ouest et le reste», qui a tant marqué la discipline anthropologique.

École des hautes études en sciences sociales Laboratoire d'anthropologie des institutions et des organisations sociales (LAIOS), Paris enric.porqueres@ehess.fr

3. Chaire d'étude comparée des sociétés africaines (1982-1998). 


\section{RÉFÉRENCES CITÉES}

\section{Bestard Camps, Joan}

1986 Casa y familia. Parentesco y reproducción doméstica en Formentera. Palma de Mallorca, Institut d'estudis baleàrics.

\section{Bourdieu, Pierre}

1972 Esquisse d'une théorie de la pratique, précédée de Trois études d'ethnologie kabyle. Genève, Droz.

\section{Delille, Gérard}

1985 Famille et propriété dans le royaume de Naples, XV'-XIXe siècles. Rome,

École française de Rome/Paris, Éd. de l'Ehess.

Gribomont, Cécile, Enric Porqueres i Gené

\& Jérôme Wilgaux, eds

2013 Incidence 9: Les incertitudes de l'inceste. Autour de l'anthropologie symbolique de Françoise Héritier. Paris, Le Félin.

\section{Héritier, Françoise}

1973 «Univers féminin et destin individuel chez les Samo", in La Notion de personne en Afrique noire. Actes du colloque international $d u$ Centre national de la recherche scientifique, Paris, 11-17 octobre 1971. Paris,

Éd. du CNRs : 243-254.

1981 L'Exercice de la parenté. Paris, Gallimard-Le Seuil («Hautes Études»).

1994 Les Deux Sours et leur mère.

Anthropologie de l'inceste. Paris, Odile Jacob.

1995 "Préface», in Enric Porqueres i Gené, Lourde Alliance. Mariage et identité chez les descendants de juifs convertis de Majorque (1435-1750). Paris, Kimé: 11-13.

\section{Lamaison, Pierre}

1979 «Les stratégies matrimoniales dans un système complexe de parenté: Ribennes en Gévaudan (1650-1830)", Annales 34 (4) : 721-743.

\section{Leach, Edmund R.}

1961 Pul Eliya, a Village in Ceylon. A Study of Land Tenure and Kinship. Cambridge, Cambridge University Press.

\section{Lévi-Strauss, Claude}

1965 "The Future of Kinship Studies", Proceedings of the Royal Anthropological Institute of Great Britain and Ireland: 13-22.

1967 Les Structures élémentaires de la parenté. $2^{\mathrm{e}}$ éd. rev. Paris-La Haye, Mouton.

1975 La Voie des masques. Genève, Skira ( Les Sentiers de la création» 24).

\section{Needham, Rodney}

1971 "Introduction", in R. Needham, ed., Rethinking Kinship and Marriage. LondonNew York, Tavistock: XV-CVIII.

\section{Porqueres i Gené, Enric}

1995 Lourde Alliance. Mariage et identité chez les descendants de juifs convertis à Majorque (1435-1750). Préf. de Françoise Héritier. Paris, Kimé ("Anthropologies»).

2001 «Le mariage qui dérange: redéfinitions de l'identité nationale basque", Ethnologie française 31 (3) : 527-536.

2006 «Parentiu i actes individuals: el temps d'una fragilitat estructurant", Revista d'etnologia de Catalunya 28: 64-69 [www.raco.cat/index.php/revistaetnologia/ article/viewFile/49482/63324].

\section{Sahlins, Marshall}

2012 What Kinship Is - And Is Not.

Chicago, Chicago University Press.

\section{Segalen, Martine}

1981 «Parenté et alliance dans les sociétés paysannes", Ethnologie française 11

(4): 307-309.

\section{Schneider, David M.}

1984 A Critique of the Study of Kinship. Ann Arbor, University of Michigan Press.

\section{Zonabend, Françoise}

1981 «Le très proche et le pas trop loin: réflexions sur l'organisation du champ matrimonial des sociétés à structures de parenté complexes ", Ethnologie française 11 (4) : 311-318. 